\title{
Collision Simulation on the Rear Protector of Truck
}

\author{
Xu Zhang ${ }^{1, a}$, Kunlun Wu ${ }^{1, b}$, Xiaohui Bai ${ }^{1, c}$, Qihe Zhang ${ }^{1, d}$, Da Song ${ }^{1, e}$ \\ and Yiwen Zhang ${ }^{1, f}$ \\ ${ }^{1}$ Harbin Engineering University, Harbin 150001, P. R. China \\ azhangxu@hrbeu.edu.cn, 'bukunlun@hrbeu.edu.cn, 'baixiaohui@hrbeu.edu.cn, 'zqh145@126.com, \\ esongda@hrbeu.edu.cn and 'zhangyiwen@hrbeu.edu.cn
}

\begin{abstract}
Keywords: car head to truck rear collision; rear protector; collision simulation
Abstract. Aimed to solve current collision problems between car head and truck rear, collision simulation of a novel type multistage rear protector is achieved, structure has been designed and optimized before. Based on conservation of energy theorem and regulations of dynamic collision test, dynamic explicit finite element analysis method is applied for investigate the device. It is shown that the peak acceleration of the car body is significantly decreased and collision safety is greatly improved when the rear protector is adopted. Through the collision simulation results of dynamic acceleration and maximum deformation, it is indicated that the novel multistage rear protector has excellent energy absorption capability, not only reducing occupant injury from collision but also improving collision compatibility during the car head to truck rear collision.
\end{abstract}

\section{Introduction}

With the improvement of living standard, more and more people drive their cars, which will cause a series of social problems, such as environmental pollution, energy crisis and traffic accidents, etc [1]. Apparently, vehicle collision is a notable problem that must be solved, especially between a car and a truck. Because crash compatibility problems are very prominent, once car head to truck rear collision occurs, it may produce a great threat to occupants, drivers and passengers. When the truck has not any rear protectors installed or the rear protectors are lack of strength enough and safety property, it will result in severe impact destruction. Therefore, the research on rear protector has great significances to improve crash compatibility and avoid traffic accidents [2].

\section{Collision test}

The main function of the rear protector is to prevent the occurrence of serious traffic accidents during car head to truck rear collisions. Based on the excellent properties of buffer and energy absorption, the rear protector can make dynamic energy from collision transformed into deformation energy, which can reduce a certain extent of damage degree on all vehicle and occupants. According to experimental regulations, the reference performances of the rear protector can be obtained by static analysis and dynamic collision test. Compared with traditional static analysis, dynamic crash test can not only offer completely maximum deformation, springback velocity and springback acceleration of moving barrier on rear protector, but also have better effects to reflect blocking ability and energy absorption capability of protector. So, dynamic crash test is chosen to assess damage barrier property of rear protector by means of dynamic explicit finite element method. Specific equipment and condition requirements can be obtained in GB11567.2-2001[3, 4].

\section{Structure model of rear protector}

The structure model of energy absorption device of the rear protector is shown in Fig.1. This is a multistage energy absorbing device. As for the first stage during collision, cross beam 4 contacts with impactive car firstly when car head to truck rear collision occurs. Generally in this stage, initial kinetic energy can be absorbed and impact speed decreases partially. Then cross beam 4 begins to squeeze energy absorption box 1 . During this second stage, collision energy is absorbed the second 
time. Under impact force, guide block 10 slides backward along guide rail 11 and squeeze energy absorption box 2 at the same time. In the third stage, plastic forming of vertical bracket 9 will further absorb the remaining energy, avoiding the occurrence of catastrophic invading collision as well. In order to ensure gradual progression and prevent energy absorption element from inclining during collisions, support beam 5 is adopted to support the structure forming along its correct way. This energy absorption device of rear protector enable impact action to conduct along a particular direction orderly and ensure each of energy absorption elements having maximized plastic deformation so that all collision energy is absorbed as much as possible.

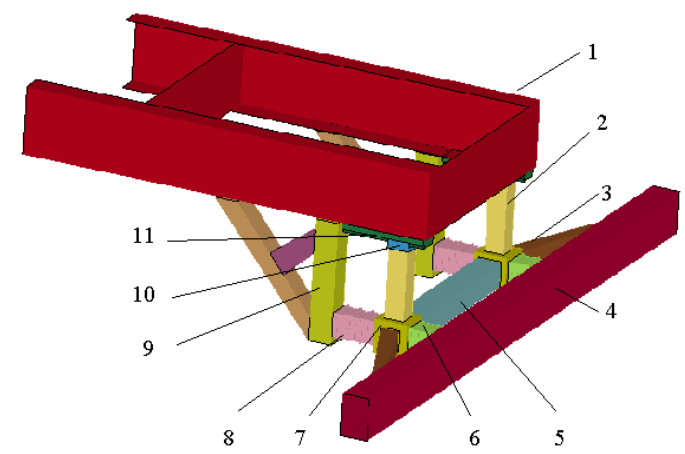

Fig.1. The structure model of energy absorption device

1. Frame 2. Cantilever 3. Inclined brace 4. Cross beam 5. Supporting beam 6 . Energy absorption box 1

7. Longitudinal beam 8 . Energy absorption box 2 9. Vertical bracket 10 . Guide block 11. Guide rail

\section{Collision simulation of rear protector}

In order to fully assess performances of the novel rear protector, collision destruction degree should be investigated deeply during car head to truck rear collision. It is known that there are a few accessories in the front part of the car being able to absorb kinetic energy by plastic deformation during collisions, such as bumper, water tank and radiator baffles [5]. So it is very necessary to achieve collision simulation to ensure the reliability of the protector and find out detailed protection properties of the rear protector. The given relative velocity between the car and the rear protector of the truck is $50 \mathrm{~km} / \mathrm{h}$. Shaded finite element model of the car head to truck rear collision is shown in Fig.2. Dynamic deformation response at different time during collision is shown in Fig.3.

It can be seen from Fig.3 that cross beam 4, energy absorption box 1, energy absorption box 2 and the front of the car have realized large plastic deformation during collision. At 5ms, the front of the car contacts with cross beam 4 firstly and then starts to squeeze other parts of rear protector. At 20ms, with the car continuing to move forward, the impact force transmits along the motion direction, energy absorption box 1 has been completely crushed, then cross beam 4 starts to bend outwardly, energy absorption box 2 and the front cover of the car also deform largely in the following extrusion process. At 40ms, energy absorption box 2 has been completely crushed, while the car is still squeezing the rear protector. At 50ms, collision energy of the car has been exhausted completely and deformation of both sides starts to springback.

According to survey report of collisions, body acceleration is typically used to evaluate casualties, the higher acceleration of the car, the more enormous impact loads suffered. Due to the intersection of the lower end of B-pillar and sill beam has much higher stiffness than other parts, the degree of collision deformation is relatively small and the deformation movement is less vulnerable from outside interference. So this intersection node is chosen to observe acceleration of the body. Acceleration curve of the lower end node of the B-pillar is shown in Fig.4. It can be seen from Fig.4 that the peak acceleration of the car body has been significantly decreased than before, and collision compatibility has been greatly improved. 


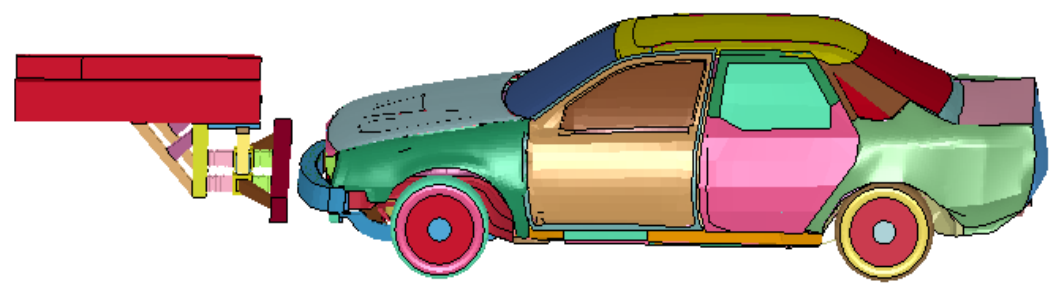

Fig.2. Finite element model

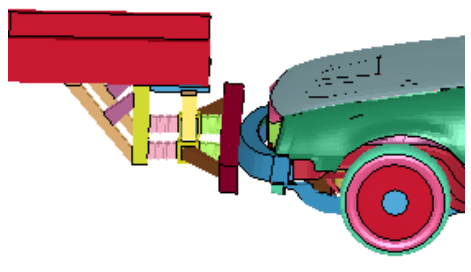

(a)

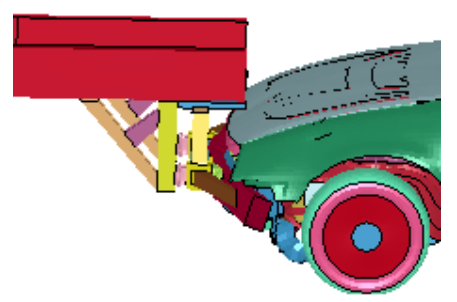

(d)

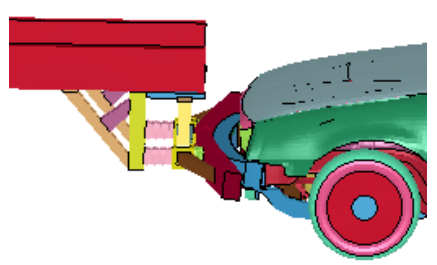

(b)

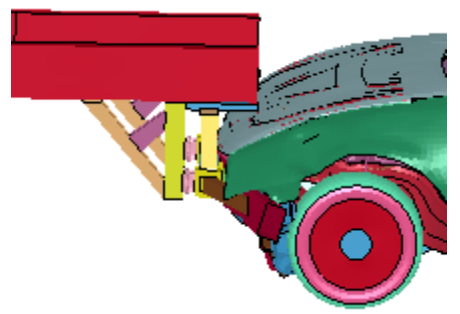

(e)

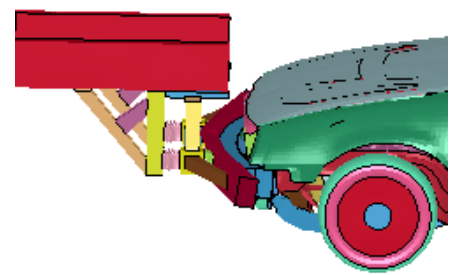

(c)

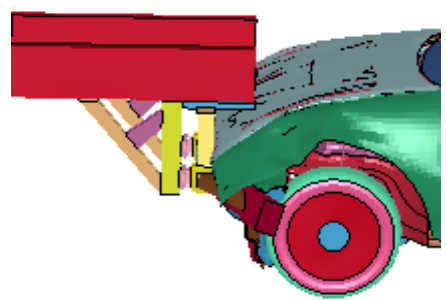

(f)

Fig.3. Dynamic deformation of the model during collision
(a ) $t=5 \mathrm{~ms}$
(b) $\mathrm{t}=15 \mathrm{~ms}$
(c) $\mathrm{t}=20 \mathrm{~ms}$
(d) $t=25 \mathrm{~ms}$
(e) $\mathrm{t}=40 \mathrm{~ms}$
(f) $\mathrm{t}=50 \mathrm{~ms}$

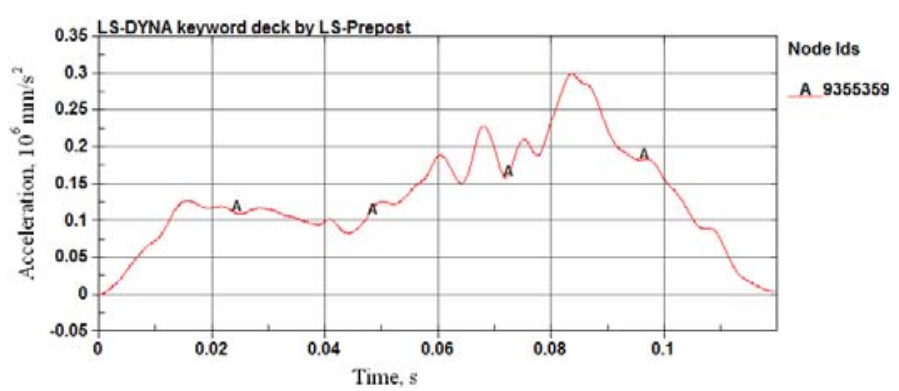

Fig.4. Acceleration of the node on the lower end of the B-pillar

\section{Analysis of energy absorption capability of rear protector}

In order to test comprehensively energy absorption capability of rear protector, relative velocity of $45 \mathrm{~km} / \mathrm{h}, 56 \mathrm{~km} / \mathrm{h}$ and $65 \mathrm{~km} / \mathrm{h}$ is selected to observe respectively deformation degree and energy absorption circumstances under different conditions. Maximum deformation of the car at $45 \mathrm{~km} / \mathrm{h}$ and 65km/h is shown in Fig.5. Acceleration of the lower end nodes of the B-pillar are shown in Fig.6.

From Fig.5 and Fig.6, it can be found that the maximum acceleration of the lower end nodes of the B pillar reaches up to $51 \mathrm{~g}$ when initial velocity is $65 \mathrm{~km} / \mathrm{h}$. Because of the high acceleration, deformation degree has become more serious. When the invading deformation increases, the rear protector even contacts with the radiator and then collides with the engine. Under other velocity conditions, the acceleration of the lower end node of the B-pillar is not high and damage degree inside the body is also acceptable. It is indicated that the novel multistage rear protector has excellent energy absorption capability, not only reducing occupant injury from collision but also improving collision compatibility during the car head to truck rear collision. 


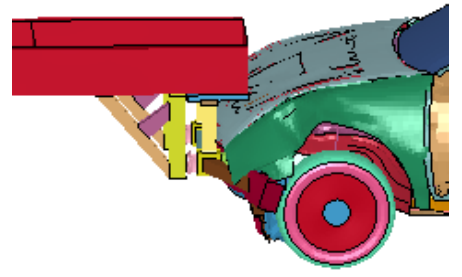

(a)

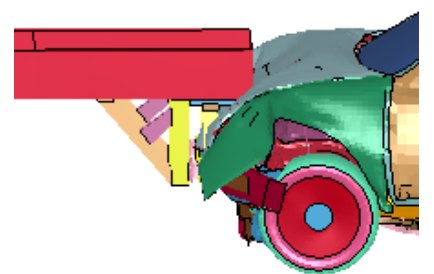

(b)

Fig.5. Maximum deformation after collision

(a) $45 \mathrm{~km} / \mathrm{h}$

(b) $65 \mathrm{~km} / \mathrm{h}$

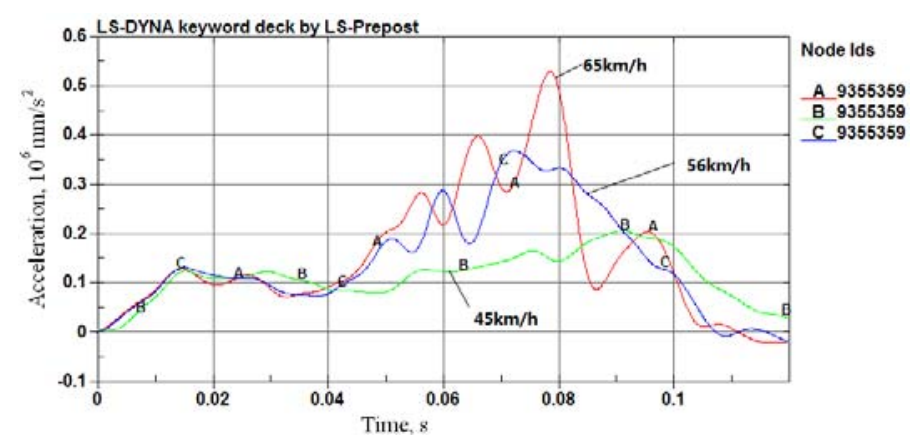

Fig.6. Acceleration of the nodes on the lower end of the B-pillar

\section{Conclusions}

To solve current problems on collision between car head and truck rear, collision simulation of a novel type multistage rear protector is achieved by dynamic explicit finite element analysis to investigate the device properties. It is shown that the peak acceleration of the car body is significantly decreased and collision safety is greatly improved when the multistage rear protector is adopted. Through the collision simulation results of dynamic acceleration and maximum deformation, it is indicated that the novel rear protector has excellent energy absorption capability, not only reducing occupant injury from collision but also improving compatibility during collision.

\section{Acknowledgements}

We gratefully acknowledge the help of Dr. Xiaodong Xing. This thesis is supported by NSFC, China (51179040).

\section{References}

[1] Chinese Automotive Technology \& Research Center. GB11567.2-2001 protection requirements of the bottom for car and trailer. China Standards Publisher (2001). (in Chinese)

[2] Hu Zhiyuan, Zeng Biqiang, Xie shugang. Analysis on automotive safety simulation based on LsDyna and HyperWorks. Tsinghua Univ. Publisher (2011). (in Chinese)

[3] Tao Yi. Study on characteristics of the energy absorber of new cars. Master Thesis. Shanghai Jiaotong Univ. (2005). (in Chinese)

[4] Wang Yudong, Jin Lei, Hong Qingquan. HyperMesh \& HyperView application skills and advanced examples. Mechanical Industry Publisher (2013). (in Chinese)

[5] Chuck A. Plaxico. Comparison of the impact performance of the G4(1W) and G4(2W) guardrail systems under CHRP Report 350 test 311 conditions. Transportation Research Record Journal of the Transportation Research Board. 2000, 1720:7-18 